\title{
SISTEMA AQUOSO BIFÁSICO: UMA ALTERNATIVA EFICIENTE PARA EXTRAÇÃo DE ÍONS
}

\author{
Maria do Carmo Hespanhol da Silva*, Luis Henrique Mendes da Silva e Fernanda Jürgensen Paggioli \\ Departamento de Química, Centro de Ciências Exatas e Tecnológicas, Universidade Federal de Viçosa, Av. P. H. Rolfs, \\ 36570-000 Viçosa - MG, Brasil \\ Jane Sélia Reis Coimbra e Luis Antonio Minim \\ Departamento de Tecnologia de Alimentos, Centro de Ciências Exatas e Tecnológicas, Universidade Federal de Viçosa, \\ Av. P. H. Rolfs, 36570-000 Viçosa - MG, Brasil
}

Recebido em 17/8/05; aceito em 9/12/05; publicado na web em 6/7/06

\begin{abstract}
AQUEOUS BIPHASIC SYSTEMS: AN EFFICIENT ALTERNATIVE FOR EXTRACTION OF IONS. Solvent extraction has been successfully applied to metal ion preconcentration and often meant the use of toxic organic diluents. However, regulatory pressure is increasingly focusing on the use and disposal of organic solvents, and thus the development of nonhazardous alternatives is important. In this review, we examine the application of aqueous biphasic systems (ABS) to extraction of ions, analyzing their potential and limitations and suggest that ABSs could be an efficient substitute for oil/water biphasic systems. ABSs are formed by mixing certain inorganic salts and water-soluble polymers, or by mixing two water-soluble polymers.
\end{abstract}

Keywords: aqueous biphasic system; metal ions; green chemistry.

\section{INTRODUÇÃO}

Nos últimos anos, as regulamentações ambientais tornaram-se mais rígidas, motivando estudos com o propósito de minimizar o descarte de resíduos nocivos à saúde humana e ao ambiente. Assim, tornou-se crescente o número de pesquisas acadêmicas e industriais voltadas para o desenvolvimento de metodologias que sejam menos poluentes, mais seguras e, principalmente, economicamente viáveis ${ }^{1}$.

Uma das metodologias aplicadas ao tratamento de resíduos é a extração líquido-líquido, pois permite o uso de grande número de diferentes solventes e agentes de extração, possibilitando que vários analitos sejam separados e pré-concentrados ${ }^{2}$. Entretanto, uma desvantagem presente na extração líquido-líquido tradicional (ELT) é o uso de solventes orgânicos que, normalmente, são tóxicos, cancerígenos e/ou inflamáveis ${ }^{3}$.

Uma alternativa promissora para substituir a ELT, baseada nos princípios da química verde ${ }^{4}$, são os sistemas aquosos bifásicos (SAB), constituídos majoritariamente por água, sendo os seus demais componentes formadores (polímeros e sais inorgânicos) não tóxicos nem inflamáveis, o que os torna um sistema de extração ambientalmente seguro. Além disso, seus constituintes são comercialmente acessíveis e de baixo custo.

Desde o final da década de 50, os SAB têm sido usados com sucesso na extração de biopartículas (como vírus, ácidos nucléicos e proteínas $)^{5-7}$, compostos inorgânicos e orgânicos ${ }^{8,9}$; e a partir da metade dos anos 80, para extração de íons ${ }^{10}$. Na Tabela 1 são apresentados diferentes SAB e algumas de suas aplicações à extração de diversos analitos.

Embora os primeiros trabalhos sobre os sistemas aquosos bifásicos tenham sido publicados a partir de 1896, por Beijerink ${ }^{21,22}$, somente em 1984 surgiu a primeira pesquisa envolvendo a aplicação dos SAB para extração de metais ${ }^{23}$.

É interessante notar que, atualmente, ainda são poucos os trabalhos realizados com a aplicação destes sistemas à partição de

*e-mail: mariacarmo@ufv.br
Tabela 1. Sistemas aquosos bifásicos e suas aplicações

\begin{tabular}{|c|c|c|c|}
\hline \multicolumn{4}{|c|}{ Sistema Aquoso Bifásico } \\
\hline Polímero 1 & Polímero 2 ou Sal & Soluto Particionado & Ref. \\
\hline $\mathrm{PEO}^{\mathrm{a}}$ & Citrato de sódio & $\alpha$-milase & 11 \\
\hline PEO & Fosfato de potássio & ZZ-cutinase-triptofano & 12 \\
\hline PEO & $\mathrm{Na}_{2} \mathrm{SO}_{4}$ & Lisozima & 13 \\
\hline PEO & Dextrana & Endo-pectinase & 14 \\
\hline PEO & $\begin{array}{c}\text { Goma de árvore } \\
\text { de caju }\end{array}$ & Tripsina & 15 \\
\hline $\begin{array}{l}\text { PEO- } \\
\text { PPO- } \\
\text { PEO }^{b}\end{array}$ & Fosfato de potássio & Insulina & 16 \\
\hline PEO & $\mathrm{K}_{2} \mathrm{CO}_{3}$ ou $\mathrm{Na}_{2} \mathrm{CO}_{3}$ & $\begin{array}{l}\mathrm{Cf}^{3+}, \mathrm{Th}^{4+}, \mathrm{Pu}^{4+}, \\
\mathrm{Ce}^{3+} \mathrm{Np}^{5+}, \mathrm{Am}^{3+}\end{array}$ & 17 \\
\hline PEO & Dextrana & $\begin{array}{c}\text { Nanosferas de } \\
\text { Au e Ag }\end{array}$ & 18 \\
\hline Dextrana & Triton X-100 & Hematita & 19 \\
\hline PEO & Dextrana & Sílica & 20 \\
\hline
\end{tabular}

${ }^{\mathrm{a}}$ Poli (óxido de etileno); ${ }^{\mathrm{b}}$ copolímero bloco:poli (óxido de etileno)poli (óxido de propileno)-poli (óxido de etileno)

íons metálicos, quando comparados com aqueles aplicados à partição de biomoléculas ${ }^{24}$. Entretanto, as inúmeras vantagens apresentadas por este novo sistema líquido-líquido têm sido um incentivo à realização de estudos sobre a partição de cátions e ânions, possibilitando sua aplicação a amostras de interesse ambiental ${ }^{25}$.

Neste artigo, portanto, é apresentada uma revisão crítica dos diversos trabalhos publicados até o momento, envolvendo a partição de íons usando SAB, mostrando a eficiência destes como técnica de extração.

\section{SISTEMA AQUOSO BIFÁSICO}

A formação espontânea de um SAB ocorre quando dois polímeros quimicamente diferentes e hidrossolúveis, ou um 
polímero e um sal inorgânico são misturados em água, desde que determinadas condições termodinâmicas críticas sejam estabelecidas, isto é, em uma faixa específica de temperatura, pressão e composição dos constituintes ${ }^{26}$.

Para um SAB produzido a partir de uma determinada composição global, geralmente definida em termos de porcentagem massa/ massa, \% (m/m), observa-se que uma das fases formadas será rica em polímero e a outra, rica em sal. Em ambas, predomina a água (Figura 1).

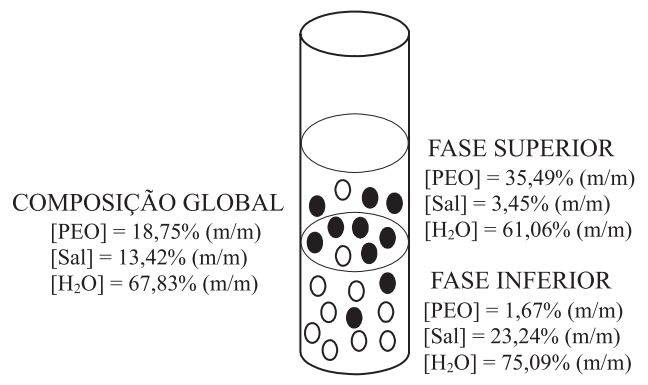

Figura 1. Composições químicas associadas a um ponto de mistura do sistema: PEO $\left(1.500 \mathrm{~g} \mathrm{~mol}^{-1}\right)+$ fosfato de potássio + água, a $25^{\circ} \mathrm{C}$ e $\mathrm{pH}=7$

A composição química das duas fases que se encontram em equilíbrio termodinâmico é geralmente representada em um diagrama de fase retangular (Figura 2). Esta representação gráfica é de grande importância para os estudos de partição, pois é utilizada inicialmente como ferramenta básica para o desenvolvimento de um processo de extração. Os dados de equilíbrio apresentados nesses diagramas estão relacionados à variação da energia livre de Gibbs do sistema, e auxiliam na compreensão dos fatores que governam a partição de um soluto qualquer nos SAB.

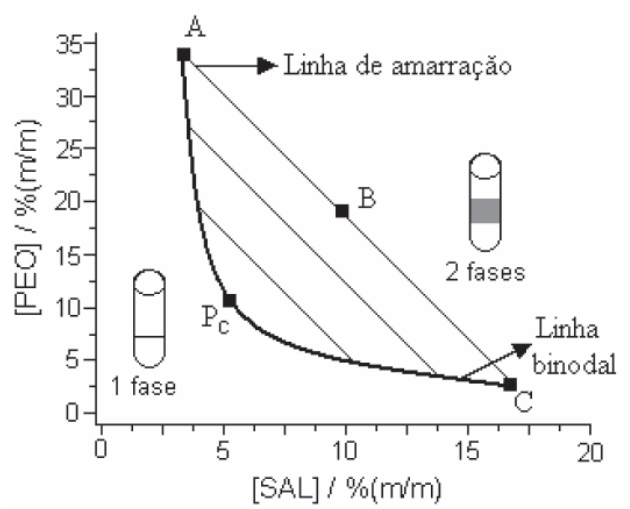

Figura 2. Diagrama de fase expresso em coordenadas retangulares de um $S A B$ formado por um polímero e um sal

Neste diagrama de fase, a abscissa representa a concentração de sal e a ordenada, a concentração do polímero presente no sistema. Dessa forma, o diagrama informa em quais composições globais o sistema é homogêneo e em quais é heterogêneo, sendo essas duas regiões separadas pela linha binodal $\left(\mathrm{AP}_{\mathrm{c}} \mathrm{C}\right)$. A posição da binodal varia de acordo com o tipo e a massa molar do polímero, a natureza química do sal, a temperatura e o $\mathrm{pH}$ do meio ${ }^{27}$. Existem diferentes métodos para obtenção da linha binodal, sendo geralmente utilizados os de titulação turbidimétrica e de análise da composição das fases.

No diagrama de fase (Figura 2) são também representadas as linhas de amarração (ex: linha $\mathrm{ABC}$ ) que, para determinada com- posição global do sistema (ponto B), fornece a concentração dos solutos nas duas fases em equilíbrio, representadas pelos pontos $\mathrm{A}$ (fase superior) e C (fase inferior). A obtenção das linhas de amarração é de grande importância, pois todas as misturas com composições globais representadas por pontos, pertencentes a uma mesma linha de amarração, fornecerão fases superiores com propriedades termodinâmicas intensivas (ex: composição) idênticas, porém com propriedades extensivas (ex: volume) diferentes. $\mathrm{O}$ mesmo princípio aplica-se às fases inferiores.

Um parâmetro termodinâmico, comumente utilizado para medir a diferença das propriedades intensivas entre as fases em equilíbrio, é o comprimento da linha de amarração (CLA). Este parâmetro é calculado em função das diferenças nas concentrações dos componentes em cada fase, conforme indicado na Equação 1.

$C L A=\left[\left(C_{P}^{S}-C_{P}^{I}\right)^{2}+\left(C_{S}^{S}-C_{S}^{I}\right)^{2}\right]^{1 / 2}$

em que $\mathrm{C}_{\mathrm{P}}{ }_{\mathrm{S}}^{\mathrm{S}} \mathrm{C}_{\mathrm{P}}{ }^{\mathrm{I}}$ são as concentrações de polímero e $\mathrm{C}_{\mathrm{S}}{ }^{\mathrm{S}}$ e $\mathrm{C}_{\mathrm{S}}{ }^{\mathrm{I}}$ são as concentrações do sal $(\%(\mathrm{~m} / \mathrm{m}))$ nas fases superior e inferior, respectivamente. À medida que o valor do CLA aumenta, torna-se maior a diferença de composição entre as fases, elevando, conseqüentemente, a eficiência na extração e/ou partição do soluto de interesse.

\section{PREPARO E USO DOS SAB PARA EXTRAÇÃO DE ÍONS}

Em geral, a metodologia para a condução de experimentos envolvendo extrações de íons metálicos com SAB é semelhante à aplicada na técnica de extração líquido-líquido tradicional. Entretanto, alguns aspectos inerentes à nova técnica merecem destaque.

O SAB é, normalmente, preparado pela mistura de alíquotas de soluções aquosas concentradas de polímero $(33,0$ a $55,0 \%$ (m/ m)) e sal (20,0 a 40,0\% (m/m)), seguida pela agitação em um vortex e posterior centrifugação. As concentrações das soluções estoque são escolhidas de modo a possibilitar a formação de um SAB com determinada composição global. Em vários estudos de partição de íons, a composição global, associada a diferentes linhas de amarração, é considerada uma propriedade termodinâmica determinante no comportamento de transferência dos solutos ${ }^{28}$.

A influência da presença de outros componentes (ex.: ácidos, bases, sais ou agentes de extração) nos SAB sobre a partição dos íons metálicos é avaliada adicionando-os em quantidades conhecidas à solução estoque do polímero ou do sal. Entretanto, para minimizar o gradiente de $\mathrm{pH}$ entre as fases, Rogers et al. ${ }^{29}$ sugeriram que seja preparada uma solução aquosa com concentração conhecida de ácido ou base, usando-a como "solvente" no preparo das soluções estoque do sal e do polímero.

Após o equilíbrio ser estabelecido, em uma dada temperatura, alíquotas das fases superior e da fase inferior são removidas para a quantificação do íon. As concentrações dos cátions e ânions em cada fase são determinadas, principalmente por métodos radiométricos ${ }^{30}$, espectrometria de absorção atômica ${ }^{31}$ e espectrometria de emissão atômica com plasma indutivamente acoplado $(\mathrm{ICP}-\mathrm{OES})^{32}$.

A partir da quantificação do metal, em ambas as fases, pode-se calcular o coeficiente de partição $\left(\mathrm{K}_{\mathrm{M}}\right)$ e a porcentagem de extração (\% E) do íon metálico, bem como o coeficiente de distribuição do metal no SAB, como definido pelas Equações 2, 3 e 4, respectivamente.

$\mathrm{K}_{\mathrm{M}}=\frac{\left[\mathrm{M}^{\mathrm{n}+}\right]_{\mathrm{S}}}{\left[\mathrm{M}^{\mathrm{n}+}\right]_{\mathrm{I}}}$ 
$\% \mathrm{E}=\frac{\left(\mathrm{n}_{\mathrm{M}^{\mathrm{n}+}}\right)_{\mathrm{S}}}{\left(\mathrm{n}_{\mathrm{M}^{\mathrm{n}+}}\right)_{\mathrm{T}}} \times 100$

$\mathrm{DM}=\frac{\left[\mathrm{C}_{\mathrm{M}}\right]_{\mathrm{S}}}{\left[\mathrm{C}_{\mathrm{M}}\right]_{\mathrm{I}}}$

em que $\left[\mathrm{M}^{\mathrm{n}+}\right]_{\mathrm{S}}$ e $\left[\mathrm{M}^{\mathrm{n}+}\right]_{\mathrm{I}}$ são as concentrações da espécie química $\mathrm{M}^{\mathrm{n}+}$ nas fases superior e inferior, respectivamente; $\left(\mathrm{n}_{\mathrm{M}^{\mathrm{n}}}\right)_{\mathrm{S}}$ indica a quantidade do íon metálico na fase superior $(S)$ e $\left(\mathrm{n}_{\mathrm{M}^{\mathrm{n}}}\right)_{\mathrm{T}}$ é a quantidade total do íon metálico adicionada ao sistema $(T)$. $\left[\mathrm{C}_{\mathrm{M}}\right]$ é a concentração total do metal nas duas fases.

O coeficiente de partição (Equação 2) é um parâmetro termodinâmico que se aplica a sistemas em que o metal se apresenta como uma única espécie química em cada fase, por ex. como um íon $\mathrm{M}^{\mathrm{n}+}$. Todavia, em muitos casos, a presença de um agente extrator, ou até mesmo do próprio ânion do sal formador do SAB, pode ocasionar a ocorrência de reações de complexação com o íon metálico, formando espécies metálicas distintas (ex: $\mathrm{M}\left(\mathrm{SO}_{4}\right)_{\mathrm{x}}^{(2 \mathrm{x}-\mathrm{n})-}, \mathrm{M}(\mathrm{X})_{\mathrm{x}}^{(\mathrm{x}-\mathrm{n})-}$ onde $\mathrm{X}^{-}$ é o agente extrator e $\mathrm{SO}_{4}^{2-}$ é o ânion proveniente do sal formador do $\mathrm{SAB}$ ), que possuem diferentes afinidades entre as fases. Isto faz com que a concentração do metal nas fases seja a soma das concentrações de todas as espécies metálicas $\left(\mathrm{C}_{\mathrm{M}}\right)$. Assim, pode-se definir o coeficiente de distribuição do metal no sistema (Equação 4).

\section{APLICAÇÃO DO SAB À PARTIÇÃO DE ÍONS METÁLICOS}

Em geral, os trabalhos aplicando os SAB na extração de íons metálicos são conduzidos de três formas: na ausência de qualquer agente extrator; na presença de um agente extrator solúvel em água e, utilizando-se um complexante do metal ligado ao polímero.

Para maior esclarecimento, o termo agente extrator ou simplesmente extrator refere-se a uma espécie química (neutra ou iônica) que é responsável pela extração do íon metálico de uma fase para a outra. Já um complexante (espécie molecular ou iônica) promove a extração do íon metálico devido à formação de um complexo metálico, que tem afinidade pela fase de interesse. Neste caso, pode-se afirmar que o complexante é um agente extrator, mas nem sempre o inverso se aplica. Exemplo disso é quando o agente extrator transfere o íon metálico para a fase de interesse, simplesmente, como seu contra íon.

Dentre as três formas de extração, a mais empregada é aquela em que se usam complexantes solúveis em água, orgânicos ou inorgânicos. Em geral, por meio desses complexantes tem-se uma melhoria na partição dos íons metálicos para a fase rica em polímero. Essa melhora é decorrente da maior interação do complexo metálico formado com os componentes presentes na fase polimérica. Dessa forma, o tipo e a concentração do complexante a ser utilizado são importantes, pois podem otimizar a extração/separação de um grupo de metais em uma amostra.

Atualmente, ainda são intensas as pesquisas para identificar diferentes componentes formadores de novos $\mathrm{SAB}^{33-36}$. Esta procura por novos sistemas ocorre porque o comportamento de partição dos íons depende de inúmeras propriedades físico-químicas associadas ao sistema (natureza química do polímero e do eletrólito, $\mathrm{pH}$, temperatura, presença de co-solutos etc.). Na Tabela 2 são apresentados alguns exemplos de $\mathrm{SAB}$, aplicados para extração de diversos íons na presença ou ausência de agentes extratores.

Nos estudos envolvendo a extração de íons metálicos, os sistemas formados por misturas de poli(óxido de etileno) (PEO) e sal inorgânico predominam em número, em relação àqueles formados pela mistura de dois polímeros. Em geral, os coeficientes de distribuição do íon metálico apresentam valores menores em sistemas constituídos por uma mistura de dois polímeros, em comparação com os obtidos em SAB formados por polímero-eletrólito. Este comportamento é observado para o íon metálico amerício que, na presença do agente extrator fosfotunguistato de potássio, apresenta no sistema $\mathrm{PEO} /$ dextrana sulfato de sódio valores de $\mathrm{D}_{\mathrm{Am}}$ de 1,5 a 2,0 $0^{37}$. Entretanto, no sistema formado por $\mathrm{PEO} /\left(\mathrm{NH}_{4}\right)_{2} \mathrm{SO}_{4}$ encontram-se valores de $\mathrm{D}_{\mathrm{Am}}$ no intervalo de 100 a $137,4^{17}$.

É interessante observar que, em certos $\mathrm{SAB}$, o sal formador do sistema também pode atuar simultaneamente como agente extrator. Este comportamento é verificado no SAB com composição global de $19,5 \%(\mathrm{~m} / \mathrm{m})$ de $\mathrm{PEO}$ e $40,5 \%(\mathrm{~m} / \mathrm{m})$ de $\mathrm{NH}_{4} \mathrm{SCN}$, em que o íon $\mathrm{Co}$ (II) e o íon $\mathrm{Fe}$ (III) apresentam valores de $\mathrm{D}_{\mathrm{M}}$ iguais a 100 e 56, respectivamente. Já no sistema 18,8\% (m/m) de PEO e 17,2\% (m/ m) de $\left(\mathrm{NH}_{4}\right)_{2} \mathrm{SO}_{4}$, a presença de $0,96 \mathrm{~mol} \mathrm{~L}^{-1} \mathrm{NH}_{4} \mathrm{SCN}$, atuando somente como agente extrator, fornece valores de $\mathrm{D}_{\mathrm{M}}$ iguais a 363 para o íon $\mathrm{Co}(\mathrm{II})$ e 100 para o íon $\mathrm{Fe}(\mathrm{III})^{38}$.

Até o momento, em poucos trabalhos tem sido avaliada a influência da massa molar média $\left(\mathrm{M}_{\mathrm{n}}\right)$ da macromolécula sobre a partição de íons, uma vez que, na maioria das pesquisas é utilizado o poli(óxido de etileno) de $\mathrm{M}_{\mathrm{n}}$ igual a $2.000 \mathrm{~g} \mathrm{~mol}^{-1}$. O emprego de polímeros de raio hidrodinâmico pequeno visa a otimização de características do sistema, como viscosidade, solubilidade do polímero e tempo de separação das fases ${ }^{40}$. Entretanto, o uso de macromoléculas de massa molar maior possibilita a formação de $\mathrm{SAB}$ contendo menores quantidades de sal e polímero e, possivelmente, diferentes comportamentos de extração.

Merece destaque a pouca aplicação de SAB formados por polímeros, que contêm monômeros diferentes do óxido de etileno ${ }^{41}$. Um exemplo disso foi a utilização do copolímero Pluronic L64, que forneceu valores de coeficiente de distribuição para o ânion pertecnatato, ligeiramente maiores que aqueles obtidos com o PEO $2.000 \mathrm{~g} \mathrm{~mol}^{-1}$ no SAB com $\left(\mathrm{NH}_{4}\right)_{2} \mathrm{SO}_{4}$ a $1,6 \mathrm{~mol} \mathrm{~L}^{-1}{ }^{42}$.

Para melhor compreensão, o polímero poli(óxido de etileno) é uma macromolécula linear que contém em sua cadeia a unidade

Tabela 2. SAB aplicados à partição de íons

\begin{tabular}{|c|c|c|c|}
\hline Componente 1 & Componente 2 & Íon & Ref. \\
\hline PEO & $\begin{array}{l}\text { Dextrana / sulfato de } \\
\text { sódio ou dextrana }\end{array}$ & Actinídeos & 37 \\
\hline PEO & $\mathrm{Na}_{2} \mathrm{SO}_{4}$ ou $\left(\mathrm{NH}_{4}\right)_{2} \mathrm{SO}_{4}$ & $\mathrm{Am}^{3+}, \mathrm{UO}_{2}^{2+}, \mathrm{Fe}^{3+}, \mathrm{Cu}^{2+}, \mathrm{Mo}^{5+}, \mathrm{Sb}^{3+}, \mathrm{Cd}^{2+}, \mathrm{Pb}^{2+}, \mathrm{Th}^{4+}, \mathrm{Lu}^{3+}$ & 17 \\
\hline PEO & ${ }^{2} \mathrm{NH}_{4} \mathrm{SCN}^{4 / 2}$ & $\mathrm{Fe}^{3+}, \mathrm{Co}^{2+}, \mathrm{Eu}^{3+}, \mathrm{Zr}^{4+}, \mathrm{Sc}^{3+}, \mathrm{Bi}^{3+}$ & 38 \\
\hline PEO & $\mathrm{K}_{2} \mathrm{CO}_{3}$ ou $\mathrm{Na}_{2} \mathrm{CO}_{3}$ & $\mathrm{Cf}^{3+}, \mathrm{Th}^{4+}, \mathrm{Pu}^{4+}, \mathrm{Bk}^{3+}, \mathrm{Ce}^{3+}, \mathrm{Np}^{5+}, \mathrm{Am}^{3+}$ & 17 \\
\hline PEO & $\mathrm{NaOH}^{3}$ ou $\mathrm{KOH}^{3}$ & $\mathrm{Ba}^{2+}, \mathrm{Cs}^{+}, \mathrm{Sr}^{2+}, \mathrm{Ca}^{2+}, \mathrm{Na}^{+}, \mathrm{Rb}^{+}$ & 17,37 \\
\hline PPO-2000 a ou PVP-K15 ${ }^{b}$ & $\left(\mathrm{NH}_{4}\right)_{2} \mathrm{SO}_{4}$ & $\mathrm{TcO}_{4}^{-}$ & 39 \\
\hline Pluronic-L64 ${ }^{c}$ & $\mathrm{~K}_{3} \mathrm{PO}_{4}$ ou $\mathrm{NaOH}$ ou $\left(\mathrm{NH}_{4}\right)_{2} \mathrm{SO}_{4}$ & $\mathrm{TcO}_{4}^{-}$ & 39 \\
\hline
\end{tabular}

${ }^{\mathrm{a}}$ Poli(óxido de propileno) de massa molar $2.000 \mathrm{~g} \mathrm{~mol}^{-1}$; ${ }^{\mathrm{b}}$ polivinilpirrolidona de massa molar $10.000 \mathrm{~g}^{\mathrm{mol}}{ }^{-1}$; ${ }^{\mathrm{c}}$ copolímero bloco de massa molar $2.900 \mathrm{~g} \mathrm{~mol}^{-1}$, constituído de poli(óxido de etileno) e poli(óxido de propileno) 
monomérica ( $\left.-\mathrm{CH}_{2}-\mathrm{CH}_{2}-\mathrm{O}-\right)$ e grupos terminais $\mathrm{R}=\mathrm{OH}$ e $\mathrm{R}$ '= H. Já o copolímero tribloco, poli(óxido de etileno)-poli(óxido de propileno)-poli(óxido de etileno), da série Pluronic é constituído por três blocos de polímero, que são designados por PEO-PPOPEO ou $(\mathrm{EO})_{\mathrm{n}}-(\mathrm{PO})_{\mathrm{m}}-(\mathrm{EO})_{\mathrm{n}}$. As estruturas químicas da macromolécula do polímero PEO e do copolímero tribloco estão representadas nas Figuras $3 \mathrm{a}$ e b, respectivamente.

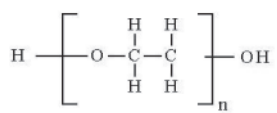

(a)

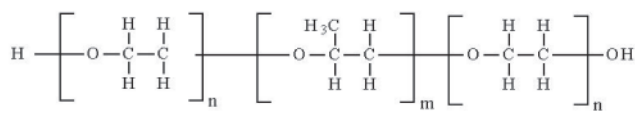

(b)

Figura 3. Estrutura química do: (a) poli(óxido de etileno); (b) copolímero tribloco poli(óxido de etileno)-poli(óxido de propileno)-poli(óxido de etileno)

Ao contrário do número reduzido de estudos acerca da influência da natureza do polímero sobre o coeficiente de distribuição dos íons metálicos, em vários trabalhos foi estudado o efeito do tipo de eletrólito sobre a distribuição de espécies carregadas nos SAB. Isto ocorreu porque é marcante a influência da natureza do eletrólito formador dos SAB sobre os valores de porcentagem de extração dos metais ${ }^{43}$.

Para comparar o efeito de diferentes componentes sobre a partição de íons, é fundamental que a análise ocorra em SAB de mesmo CLA. Isto se deve ao fato de alguns compostos produzirem SAB com distintas linhas binodais e, conseqüentemente, uma mesma composição global pode produzir fases com diferentes propriedades termodinâmicas intensivas, causadas apenas pela diferença em composição química (CLA). Este efeito pode ser observado na Figura 4, onde a eficiência dos eletrólitos em induzir a separação de fase segue a seguinte ordem: $\left(\mathrm{NH}_{4}\right)_{2} \mathrm{SO}_{4}<\mathrm{K}_{2} \mathrm{CO}_{3}<\mathrm{NaOH}$. Dessa forma, para uma mesma quantidade de PEO adicionada ao sistema, menor quantidade do sal $\mathrm{NaOH}$ é requerida para que ocorra a formação do SAB.

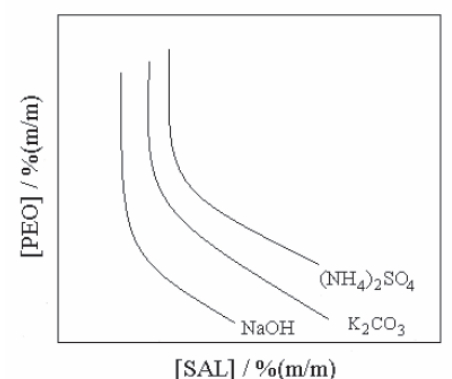

Figura 4. Diagrama de fase de três SAB constituídos por PEO $2.000 \mathrm{~g} \mathrm{~mol}^{-1}$ e diferentes eletrólitos

Assim, deve-se tomar cuidado ao concluir, por ex., que a partição dos íons $\mathrm{Sr}^{2+}$ e $\mathrm{Cs}^{+}$, no sistema $\mathrm{PEO} / \mathrm{NaOH}$ e na ausência de qualquer agente extrator, é maior que nos sistemas $\mathrm{PEO} /\left(\mathrm{NH}_{4}\right)_{2} \mathrm{SO}_{4}$ ou $\mathrm{PEO} /$ $\mathrm{K}_{2} \mathrm{CO}_{3}$. Esta conclusão será verdadeira apenas se os valores de $\mathrm{D}_{\mathrm{M}}$ forem comparados entre sistemas com o mesmo valor de CLA.

Apesar do conhecimento de que a temperatura pode influenciar o valor de $\mathrm{D}_{\mathrm{M}}$ nos $\mathrm{SAB}^{44,45}$, as extrações são conduzidas geralmente sem a termostatização do sistema e em temperatura ambiente $\left(\cong 25^{\circ} \mathrm{C}\right)$.

\section{Extração de íons em SAB sem o uso de agentes extratores}

Em geral, cátions tendem a se transferirem preferencialmente para a fase rica em sal. Por ex., os íons $\mathrm{Ce}^{3+}, \mathrm{Eu}^{3+}, \mathrm{Cr}^{3+} \mathrm{e}^{3+}$ possuem coeficientes de distribuição que variam desde 0,06 até $0,71 \mathrm{em}$ um SAB formado por $15,0 \%(\mathrm{~m} / \mathrm{m})$ de PEO $2.000 \mathrm{~g} \mathrm{~mol}^{-1}$ e $14,4 \%(\mathrm{~m} / \mathrm{m})$ de $\left(\mathrm{NH}_{4}\right)_{2} \mathrm{SO}_{4}$, com pH variando entre 2,5 e 4,023. Porém, alguns ânions possuem coeficientes de distribuição maiores que um, indicando uma transferência preferencial para a fase rica em polímero. No SAB descrito no parágrafo anterior, os ânions $\mathrm{Br}^{-}, \mathrm{SCN}^{-}$e $\mathrm{I}^{-}$possuem coeficiente de distribuição igual a 3,16; 5 e 10, respectivamente. Entretanto, alguns ânions se concentram na fase salina como, por ex., o ânion $\mathrm{PO}_{4}^{3-}$ com coeficiente de distribuição igual a $0,25^{23}$.

É interessante destacar que os cátions $\mathrm{Cd}^{2+}, \mathrm{Zn}^{2+}, \mathrm{Cu}^{2+} \mathrm{e} \mathrm{Co}^{2+}$ no SAB PEO $2.000 \mathrm{~g} \mathrm{~mol}^{-1} /\left(\mathrm{NH}_{4}\right)_{2} \mathrm{SO}_{4}$ apresentam comportamento de distribuição fortemente dependente dos valores de $\mathrm{pH}$, da concentração do metal adicionado e da composição global do sistema ${ }^{46}$. A alteração desses parâmetros pode ser utilizada para a extração seletiva e separação desses íons metálicos presentes em matrizes complexas. $\mathrm{Na}$ Tabela 3 é apresentada a influência da presença de co-solutos (ácidos, bases ou sais), em determinada concentração, sobre a extração de alguns metais. Estes dados foram obtidos em SAB 40,0\% (m/ m) de PEO $2.000 \mathrm{~g} \mathrm{~mol}^{-1} \mathrm{com} 40,0 \%(\mathrm{~m} / \mathrm{m})$ de $\left(\mathrm{NH}_{4}\right)_{2} \mathrm{SO}_{4}{ }^{47}$.

Tabela 3. Coeficiente de distribuição do íon metálico na ausência de complexante em um SAB formado por $40,0 \%(\mathrm{~m} / \mathrm{m})$ PEO 2.000 g mol ${ }^{-1}$ e $40,0 \%(\mathrm{~m} / \mathrm{m})\left(\mathrm{NH}_{4}\right)_{2} \mathrm{SO}_{4}$ a $25^{\circ} \mathrm{C}$

\begin{tabular}{lcccc}
\hline Íon & & \multicolumn{3}{c}{$\mathrm{D}_{\mathrm{M}}$} \\
& - & $\mathrm{HNO}_{3}$ & $\mathrm{H}_{2} \mathrm{SO}_{4}$ & $\mathrm{NH}_{4} \mathrm{NO}_{3}$ \\
\hline $\mathrm{Cd}^{2+}$ & 0,066 & $0,150^{\mathrm{a}}$ & $0,220^{\mathrm{a}}$ & - \\
$\mathrm{Hg}^{2+}$ & 0,260 & $10^{\mathrm{a}}$ & $12^{\mathrm{a}}$ & - \\
$\mathrm{Bi}^{3+}$ & 0,050 & $0,037^{\mathrm{a}}$ & $0,043^{\mathrm{a}}$ & - \\
$\mathrm{Pb}^{2+}$ & 0,010 & $0,320^{\mathrm{a}}$ & $0,190^{\mathrm{a}}$ & - \\
$\mathrm{UO}_{2}^{2+}$ & 0,085 & $0,039^{\mathrm{b}}$ & $0,065^{\mathrm{b}}$ & $0,019^{\mathrm{b}}$ \\
$\mathrm{Pu}^{4+}$ & 0,045 & $0,013^{\mathrm{b}}$ & $0,018^{\mathrm{b}}$ & $0,013^{\mathrm{b}}$ \\
$\mathrm{Th}^{4+}$ & 0,026 & $0,015^{\mathrm{b}}$ & $0,016^{\mathrm{b}}$ & $0,0068^{\mathrm{b}}$ \\
$\mathrm{Am}^{3+}$ & 0,0096 & $0,0083^{\mathrm{b}}$ & $0,011^{\mathrm{b}}$ & $0,0041^{\mathrm{b}}$
\end{tabular}

a A concentração do ácido em estudo é de 2,00 mol L-1 em ambas as fases do $\mathrm{SAB}$; ${ }^{\mathrm{b}}$ presença de 2,00 mol L-1 de ácido ou $\mathrm{NH}_{4} \mathrm{NO}_{3}$ na solução estoque salina formadora do $\mathrm{SAB}$.

Surpreendentemente, o íon $\mathrm{Hg}(\mathrm{II})$ sofre forte influência quando estão presentes os ácidos sulfúrico ou nítrico, apresentando valores de $\mathrm{D}_{\mathrm{Hg}}$ que variam entre 0,26 e 12,00. O comportamento específico deste íon ainda não é completamente compreendido, requerendo, portanto, maior investigação.

Ao contrário dos demais cátions e ânions, o íon pertecnatato, $\mathrm{TcO}_{4}^{-}$, apresenta forte afinidade pela fase rica em polímero $\left(10^{-3}<\right.$ $\left.\mathrm{D}_{\mathrm{TcO}_{4}}<4000\right)$, não havendo, portanto, necessidade de usar agentes extratores para uma partição quantitativa ${ }^{48}$. A importância dos estudos realizados, envolvendo a partição desse ânion $\left(\mathrm{TcO}_{4}^{-}\right)$em SAB, está relacionada ao interesse de: promover a purificação de amostras de ${ }^{99} \mathrm{Tc}$ para sua reutilização em procedimentos envolvidos na medicina nuclear; remover o ${ }^{99} \mathrm{TcO}_{4}^{-}$em resíduos nucleares altamente alcalinos, no qual a alta meia-vida $\left(\mathrm{t}_{1 / 2}=2,12 \times 10^{5}\right.$ anos $)$ e alta mobilidade ambiental do ${ }^{99} \mathrm{Tc}$ apresenta problemas para longos tempos de estocagem e, separar os radioisótopos de metais do grupo 1 e 2 presentes em grande quantidade em resíduos alcalinos, uma vez que estes interferem, significativamente, no processo de remoção seletiva de outros componentes, como pertecnatato e actinídeos $^{49,50}$. 
De fato, o único ânion com valores de $\mathrm{D}_{\mathrm{M}}$ acima de 20 , podendo chegar até valores superiores a 1.000 , é o ânion pertecnatato. $\mathrm{O}$ aumento da massa molar do polímero ${ }^{51,52}$ provoca incremento no valor do $\mathrm{D}_{\mathrm{TcO}_{4}}$ - e o aumento na concentração do sal ou do polímero favorece a transferência do ânion para a fase rica em polímero ${ }^{53}$. Estes resultados expressam o efeito clássico do aumento do CLA sobre a partição de solutos nos SAB. Aumentar a diferença de composição entre as fases resultará, sempre, no incremento da diferença de propriedades termodinâmicas intensivas entre essas fases e, conseqüentemente, em aumento no coeficiente de partição ${ }^{54}$.

A natureza química do polímero também afeta a distribuição do ânion $\mathrm{TcO}_{4}$. Para $\mathrm{SAB}$ formados por polímero/ $\left(\mathrm{NH}_{4}\right)_{2} \mathrm{SO}_{4}$, observou-se a seguinte ordem crescente para os valores de $\mathrm{D}_{\mathrm{TcO}_{4}}: \mathrm{PPO}$ $2.000 \mathrm{~g} \mathrm{~mol}^{-1}<$ PVP-K15 < PEOs < Pluronic L64. Estes resultados demonstram que a hidrofobicidade do polímero pode ser utilizada para otimizar a partição deste ânion.

Em relação à influência da natureza do eletrólito formador das fases sobre a partição do íon $\mathrm{TcO}_{4}^{-}$, observa-se uma contribuição tanto do ânion quanto do cátion. Entretanto, é menor o efeito causado pela espécie positiva. Rogers e colaboradores ${ }^{30}$ relataram a existência de uma relação linear entre a energia livre de hidratação do cátion ou do ânion formador dos $\mathrm{SAB}$ e os valores de $\mathrm{D}_{\mathrm{TcO}_{4}}$. Esta dependência da partição do ânion com parâmetros termodinâmicos de solvatação fornece uma forma simples de predizer a distribuição do $\mathrm{TcO}_{4}^{-}$em diferentes $\mathrm{SAB}$.

Merece destaque, também, a relação linear observada entre $\mathrm{D}_{\mathrm{TcO}_{4}}$ e a energia livre de Gibbs de hidratação total associada à solução estoque salina $\left(\Delta_{\text {hid }} \mathrm{G} \text { do cátion }+\Delta_{\text {hid }} \mathrm{G} \text { do ânion }\right)^{55,56}$. Porém, sabese que a presença de outras espécies iônicas em efluentes industriais pode afetar a partição do íon pertecnatato, em determinado sistema aquoso bifásico.

Interessantemente, o aumento na concentração de $\mathrm{H}_{2} \mathrm{SO}_{4}$, de 0,0010 para 2,0 mol L-1 diminui o valor de $\mathrm{D}_{\mathrm{TcO}_{4}}$ de 450 para 31 . O aumento na concentração de certos haletos leva, também, à redução do valor de $\mathrm{D}_{\mathrm{TcO}_{4}^{-}}$, sendo observada a seguinte ordem: $\mathrm{Cl}^{-}<\mathrm{Br}^{-}$ $<\mathrm{I}^{-}$. Entretanto, a adição do ânion fluoreto promove um incremento na concentração do íon pertecnatato na fase rica em polímero ${ }^{57}$.

A similaridade entre os íons $\mathrm{TcO}_{4}^{-}$e $\mathrm{ReO}_{4}^{-}$tem possibilitado o desenvolvimento de técnicas eficientes de extração, utilizando SAB para separação dos íons $\mathrm{ReO}_{4}^{-} / \mathrm{WO}_{4}^{2-}$ e $\mathrm{ReO}_{4}^{-} / \mathrm{MoO}_{4}{ }^{2-}$ em amostras de interesse radiofarmacêutico e hidrometalúrgico, respectivamente ${ }^{58}$.

Existe, também, um forte interesse na remoção de alguns actinídeos $\left(\mathrm{Am}^{3+}, \mathrm{Pu}^{4+}, \mathrm{Th}^{4+}\right.$ e $\left.\mathrm{UO}_{2}{ }^{2+}\right)$ em resíduos industriais devido a alta toxicidade, mobilidade ambiental e radioatividade, inerentes a estes íons ${ }^{59}$. Entretanto, a obtenção de valores de $\mathrm{D}_{\mathrm{M}}$ menores do que 1,0 , para esses actinídeos nos SAB estudados ${ }^{60}$, mostrou a necessidade do emprego de um agente extrator, a fim de conseguir sua remoção nos resíduos alcalinos ${ }^{61,62}$.

Em geral, conclui-se que na ausência de um agente extrator, com exceção do íon pertecnatato, os demais íons metálicos apresentaram baixos coeficientes de distribuição nos SAB estudados, devido principalmente à alta afinidade destes pela fase rica em sal. Entretanto, este comportamento de partição pode ser alterado modificando-se o tipo de ânion do sal formador do SAB ou a natureza química do polímero, assim como com a presença de co-solutos (ex: ácidos).

\section{Extração de íons em SAB na presença de um agente extrator solúvel em água}

A baixa extração dos íons metálicos da fase salina para a fase rica em polímero levou ao estudo da influência de agentes extratores solúveis em água sobre o coeficiente de distribuição do íon metáli- co. Na Tabela 4 são apresentados alguns agentes extratores orgânicos e inorgânicos (solúveis em água) utilizados no estudo da partição de diversos íons metálicos em SAB.

Tabela 4. Tipos de complexantes solúveis em água utilizados para partição de íons metálicos em SAB

\begin{tabular}{|c|c|c|}
\hline Extrator & Íons Metálicos Estudados & Ref. \\
\hline DHPMIAA $^{\mathrm{a}}$ & $\mathrm{Eu}^{3+} \mathrm{e} \mathrm{Am}^{3+}$ & 46 \\
\hline $\mathrm{AC}^{\mathrm{b}}$ & $\begin{array}{c}\mathrm{Th}^{4+}, \mathrm{UO}_{2}^{2+}, \mathrm{Pu}^{4+}, \mathrm{Am}^{3+} \mathrm{Eu}^{3+}, \mathrm{Cm}^{3+} \\
\mathrm{Bk}^{3+}, \mathrm{Cf}^{3+}, \mathrm{Es}^{3+}, \mathrm{Ce}^{3+}{\mathrm{e} \mathrm{Np}^{5+}}^{5+}\end{array}$ & $\begin{array}{c}17,60 \\
63-66\end{array}$ \\
\hline 18-coroa-6 & $\begin{array}{c}\mathrm{Na}^{+}, \mathrm{Rb}^{+}, \mathrm{Cs}^{+}, \mathrm{Ca}^{2+}, \mathrm{Sr}^{2+} \\
\mathrm{Ba}^{2+}, \mathrm{Th}^{4+}, \mathrm{UO}_{2}^{2+}, \mathrm{Pu}^{4+} \mathrm{Am}^{3+}\end{array}$ & $47,55,67$ \\
\hline 15 -coroa-5 & $\begin{array}{c}\mathrm{Na}^{+}, \mathrm{Rb}^{+}, \mathrm{Cs}^{+}, \mathrm{Sr}^{2+}, \mathrm{Am}^{3+} \\
\mathrm{Pu}^{4+}, \mathrm{Th}^{4+}, \mathrm{UO}_{2}^{2+}{\mathrm{e} \mathrm{Ba}^{2+}}^{2+}\end{array}$ & $47,55,67$ \\
\hline 12-coroa-4 & $\mathrm{Sr}^{2+} \mathrm{e} \mathrm{Cs}^{1+}$ & 67 \\
\hline Sal de nitroso $\mathrm{R}$ & $\mathrm{Fe}^{3+}$ & 23 \\
\hline $\mathrm{NH}_{4} \mathrm{SCN}$ & $\begin{array}{c}\mathrm{Mo}^{5+}, \mathrm{Fe}^{3+}, \mathrm{Cu}^{2+}, \mathrm{In}^{3+}, \mathrm{Zn}^{2+}, \mathrm{Ga}^{3+} \\
\mathrm{Co}^{2+}, \mathrm{Mn}^{2+}, \mathrm{Ni}^{2+}, \mathrm{Cd}^{2+}, \mathrm{Hf}^{4+}, \mathrm{Sc}^{3+}{\mathrm{e} \mathrm{Li}^{1+}}^{1+}\end{array}$ & 23,38 \\
\hline Iodeto & $\begin{array}{c}\mathrm{In}^{3+}, \mathrm{Bi}^{3+}, \mathrm{Cd}^{2+}, \mathrm{Cu}^{1+}, \mathrm{Sb}^{3+}, \mathrm{Tl}^{3+}, \mathrm{Zn}^{2+} \\
\mathrm{Pb}^{2+}, \mathrm{Mn}^{2+}, \mathrm{Fe}^{3+}, \mathrm{Ni}^{2+}, \mathrm{Cu}^{2+}, \mathrm{Li}^{1+}{\mathrm{e} \mathrm{Hg}^{2+}}^{2+}\end{array}$ & $\begin{array}{r}23,38,41 \\
47,30,64\end{array}$ \\
\hline Fluoreto & $\mathrm{Nb}^{+5}, \mathrm{Tl}^{+}, \mathrm{Hg}^{2+}, \mathrm{Pb}^{2+}, \mathrm{Bi}^{3+}, \mathrm{Cd}^{2+}$ & 29 \\
\hline $\mathrm{NH}_{3}$ & $\mathrm{Cr}^{6+}$ & 32 \\
\hline
\end{tabular}

aácido 1,3-diidroxifenil-metilaminodiacético; 'balizarina complexona.

Nota-se o uso predominante de complexantes orgânicos para a extração dos metais alcalinos, alcalinos terrosos e actinídeos, enquanto agentes extratores inorgânicos são usados para a transferência dos metais de transição. Para extração e/ou separação de certos íons metálicos, são eficientes os agentes extratores orgânicos solúveis em água, por apresentarem partição quantitativa para a fase rica em polímero e elevada constante de estabilidade do complexo formado. As moléculas desses complexantes possuem, geralmente, anéis aromáticos e grupos funcionais sulfônicos ${ }^{8,68}$.

A presença de estruturas aromáticas nessas moléculas complexantes aumenta o coeficiente de partição destas. Por ex., o azul de metiltimol (AM), alaranjado de xilenol (AX) e arsenazo III possuem valores de $\mathrm{K}>100$, enquanto os de alguns complexantes alifáticos possuem valores de $\mathrm{K}$ inferiores a $\mathrm{um}^{69}$.

Esta diferença na eficiência extrativa de cada complexante pode ser utilizada para otimizar a separação de um grupo de íons metálicos. Por ex., o uso simultâneo de dois agentes extratores, como AX e ácido hidroxietildifosfônico (HEDPA), leva a fatores de separação entre 4,5 e 5,6, para os íons $\mathrm{Am}^{3+} \mathrm{e} \mathrm{Eu}^{3+}$ no sistema PEO 2.000 $\mathrm{g} \mathrm{mol}^{-1} / \mathrm{K}_{2} \mathrm{CO}_{3}$, com o HEDPA atuando como um agente mascarante do íon $\mathrm{Eu}^{3+63}$.

Na Tabela 5 são apresentados vários fatores de separação encontrados para alguns íons metálicos, na presença de determinado extrator.

É interessante observar que, na presença do agente extrator, o íon metálico pode ser retirado da fase rica em PEO, controlando-se apenas o $\mathrm{pH}$ das fases. Verifica-se que $85,2 \%$ do íon amerício, extraído por arsenazo III, são retornados para a fase rica em sal, quando o valor do $\mathrm{pH}$ das fases é ajustado a 2,4 no sistema PEO $2.000 \mathrm{~g} \mathrm{~mol}^{-1} /\left(\mathrm{NH}_{4}\right)_{2} \mathrm{SO}_{4}{ }^{70}$.

Alguns agentes extratores, como arsenazo III, alizarina complexona (AC), AX e AM, que mostram distribuição quantitativa para a fase rica em PEO têm sido utilizados para estudos sobre a extração de actinídeos, lantanídeos e certos metais de transição em sistemas formados por PEO e sal (sulfato, carbonato ou fosfato).

Molochnikova et al. $^{71}$ estudaram o efeito do arsenazo III sobre a partição de íons metálicos $\mathrm{Am}^{3+}, \mathrm{Cm}^{3+}, \mathrm{Bk}^{3+}, \mathrm{Cf}^{3+}, \mathrm{Pu}^{4+}, \mathrm{U}^{4+}, \mathrm{Th}^{4+}, \mathrm{Eu}^{3+}$ e $\mathrm{Ce}^{3+}$ no SAB composto de $15,0 \%(\mathrm{~m} / \mathrm{m})$ de PEO $2.000 \mathrm{~g} \mathrm{~mol}^{-1} \mathrm{e}$ 
Tabela 5. Fatores de separação encontrados para íons metálicos nos SAB

\begin{tabular}{lccccc}
\hline SAB & Metais & \multicolumn{4}{c}{ Fator de Separação (Fs) } \\
& & Arsenazo III & $\mathrm{AM}^{\mathrm{b}}$ & $\mathrm{AX}^{\mathrm{c}}$ & $\mathrm{AC}^{\mathrm{d}}$ \\
\hline $\mathrm{PEO} \mathrm{e}$ & $\mathrm{Th}^{4+} / \mathrm{Eu}^{3+}$ & $>100^{\mathrm{e}}$ & - & - & - \\
$\left(\mathrm{NH}_{4}\right)_{2} \mathrm{SO}_{4}$ & $\mathrm{Pu}^{4+} / \mathrm{Ce}^{3+}$ & $57,1^{\mathrm{f}}$ & - & - & - \\
& $\mathrm{Pu}^{4+} / \mathrm{Am}^{3+}$ & $55,2^{\mathrm{f}}$ & - & - & - \\
$\mathrm{PEO} \mathrm{e}$ & $\mathrm{ETP}^{\mathrm{g}} / \mathrm{Np}$ & - & 61,9 & 679 & 487 \\
$\mathrm{~K}_{2} \mathrm{CO}_{3}$ & $\mathrm{Eu}, \mathrm{Ce} / \mathrm{Np}$ & - & - & 168 & 221 \\
& $\mathrm{Eu}, \mathrm{Ce} / \mathrm{U}$ & - & - & 884 & 607 \\
& $\mathrm{Eu}, \mathrm{Ce} / \mathrm{Pu}$ & - & & 323 & - \\
\hline
\end{tabular}

${ }^{\mathrm{a}}$ Arsenazo III $\left(1 \times 10^{-3} \mathrm{~mol} \mathrm{~L}^{-1}\right)$; ${ }^{\mathrm{b}} \mathrm{AM}\left(0,02 \mathrm{~mol} \mathrm{~L}^{-1}\right)$; ${ }^{\mathrm{c}} \mathrm{AX}(0,02 \mathrm{~mol}$ $\left.\mathrm{L}^{-1}\right)$; dalizarina complexona a $0,02 \mathrm{~mol} \mathrm{~L}{ }^{-1} ;{ }^{\mathrm{e}} \mathrm{p} \mathrm{H}=1,5 ;{ }^{\mathrm{f}} \mathrm{pH}=2,2$; gelementos transplutônicos (ETP): Es, Cf, Bk, Cm e Am.

$14,4 \%(\mathrm{~m} / \mathrm{m})$ de $\left(\mathrm{NH}_{4}\right)_{2} \mathrm{SO}_{4}$. Conforme apresentado anteriormente, na ausência do extrator, estes cátions tendem a permanecer na fase rica em sal, sendo os íons $\mathrm{Cf}^{3+}, \mathrm{Eu}^{3+}, \mathrm{Ce}^{3+} \mathrm{e} \mathrm{Th}^{4+}$ transferidos para a fase rica em polímero com $\% \mathrm{E}$ menor que $10,0 \%$.

$\mathrm{Na}$ presença do extrator arsenazo III $\left(1,0 \times 10^{-3} \mathrm{~mol} \mathrm{~L}^{-1}\right)$ observou-se a extração quantitativa dos actinídeos e lantanídeos para a fase polimérica, com valores de $15<\mathrm{D}_{\mathrm{M}}<317$ no intervalo de $\mathrm{pH}$ entre 3,5 e 4,5. A ordem de partição entre os actinídeos trivalentes foi $\mathrm{Am}<\mathrm{Cm}<\mathrm{Cf}<\mathrm{Bk}$ em $\mathrm{pH} 3,2$. O ajuste do $\mathrm{pH}$ em torno de 2 reduziu os valores de $\mathrm{D}_{\mathrm{M}}$ de 100 a aproximadamente 10,0 para os elementos $\mathrm{Th}^{4+} \mathrm{e} \mathrm{Pu}^{4+}$, e para menos que 1,0 para os íons $\mathrm{Eu}^{3+}, \mathrm{Ce}^{3+}$, $\mathrm{Am}^{3+}, \mathrm{Cm}^{3+}, \mathrm{Bk}^{3+}, \mathrm{Cf}^{3+}$ e $\mathrm{U}^{4+}$.

A mudança do tipo de sal formador do $\mathrm{SAB}$, de sulfato para carbonato, leva a uma extração insignificante dos actinídeos $\mathrm{Am}^{3+}, \mathrm{Pu}^{4+}$, $\mathrm{Th}^{4+} \mathrm{e} \mathrm{UO}_{2}^{2+}$ para a fase rica em PEO, mesmo com o extrator arsenazo III no sistema 40,0\% (m/m) de PEO $2.000 \mathrm{~g} \mathrm{~mol}^{-1}$ e 40,0\% (m/m) de $\mathrm{K}_{2} \mathrm{CO}_{3}{ }^{62}$. Entretanto, obtém-se extração efetiva dos actinídeos e lantanídeos quando se utilizam os extratores $\mathrm{AX}, \mathrm{AC}$ e $\mathrm{AM}^{71}$.

O 4-sulfônico-calix-6-arense foi outro agente extrator estudado para a partição dos íons metálicos $\left(\mathrm{Na}^{+}, \mathrm{Cs}^{+}, \mathrm{Sr}^{2+}, \mathrm{Ba}^{2+}, \mathrm{Co}^{2+}, \mathrm{Cd}^{2+} \mathrm{e}\right.$ $\left.\mathrm{UO}_{2}{ }^{2+}\right)$ no SAB PEO $2.000 \mathrm{~g} \mathrm{~mol}^{-1} /\left(\mathrm{NH}_{4}\right)_{2} \mathrm{SO}_{4}$, em um meio fracamente ácido ${ }^{72}$. Este complexante não foi efetivo na extração desses íons.

Os complexantes éter 12-coroa-4, éter 15-coroa-5 e éter 18-coroa-6 foram também aplicados à extração dos íons actinídeos, alcalinos e alcalinos terrosos em SAB formados por polímeros e eletrólitos ${ }^{73,74}$. $\mathrm{O}$ uso desses extratores foi motivado por sua disponibilidade comercial e seletividade para os metais dos grupos I e II. Entretanto, esses complexantes transferem-se muito pouco para a fase rica em polímero, razão pela qual fornecem baixos valores para a extração de diversos íons.

É interessante observar que, para os íons $\mathrm{Am}^{3+}, \mathrm{Pu}^{4+}, \mathrm{Pu}^{6+}, \mathrm{Th}^{4+}$, $\mathrm{UO}_{2}^{2+}, \mathrm{Na}^{+}, \mathrm{Ca}^{2+}, \mathrm{Rb}^{+}, \mathrm{Sr}^{2+}, \mathrm{Cs}^{+} \mathrm{e} \mathrm{Ba}^{2+}$ o extrator éter 18-coroa- 6 foi mais eficiente na extração que o complexante éter 15 -coroa- $5^{55}$. A eficiência desses extratores pode ser aumentada pela adição de $\mathrm{NaNO}_{3}\left(2,0 \mathrm{~mol} \mathrm{~L}^{-1}\right)$ na solução estoque salina do sal formador do sistema. Variáveis como a concentração de ácidos adicionados e a natureza do eletrólito formador dos SAB também influenciam a extração de íons utilizando éter coroa.

Objetivando a transferência quantitativa de íons metálicos da fase salina para a polimérica, foram utilizados alguns complexantes inorgânicos. Em destaque, citam-se tiocianato, $\mathrm{K}_{10} \mathrm{P}_{2} \mathrm{~W}_{17} \mathrm{O}_{61} \cdot \mathrm{nH}_{2} \mathrm{O}$, $\mathrm{H}_{3} \mathrm{PO}_{4} \cdot 12 \mathrm{WO}_{3} \cdot 10 \mathrm{H}_{2} \mathrm{O}$ e haletos $\left(\mathrm{F}^{-}, \mathrm{Cl}^{-}, \mathrm{Br}^{-}\right.$e I- $)$. Esses extratores formam complexos aniônicos com os íons metálicos que, juntamente com um contra-íon (ex: cátion do sal formador de fase), podem ser simultaneamente transferidos para fase polimérica ${ }^{25}$.
Verificou-se que o emprego desses complexantes inorgânicos intensificou a influência da natureza do eletrólito formador dos SAB, do $\mathrm{pH}$ do sistema, da temperatura e da espécie química empregada como extrator sobre o coeficiente de distribuição do metal. Um exemplo disso é a natureza química do agente extrator, onde o emprego de haletos seguirá, sempre, a seguinte ordem de eficiência: $\mathrm{I}^{-}>\mathrm{Br}^{-}$ $>\mathrm{Cl}^{-}>\mathrm{F}^{-29}$. Esta seletividade tem sido correlacionada com as propriedades físico-químicas associadas ao complexo: constante de formação $\left(\mathrm{K}_{\mathrm{f}}\right)$, densidade de carga $\left(d_{\left.\mathrm{Mx}_{4}{ }^{2}\right)}\right)$ e energia livre de Gibbs de hidratação $\left(\Delta_{\text {hid }} \mathrm{G}\right)^{29}$. Entretanto, aindã não são conhecidas as forças motrizes responsáveis por essa extração seletiva. Observa-se que um aumento na densidade de carga do complexo metálico reduz sua migração para a fase rica em polímero, enquanto a formação de complexos mais estáveis facilita sua transferência da fase inferior para a fase superior ${ }^{75}$

Em estudos envolvendo a extração dos íons $\mathrm{Tl}^{+}, \mathrm{Pb}^{2+}, \mathrm{Bi}^{3+} \mathrm{e}$ $\mathrm{Cd}^{2+}$ em SAB formados por 40,0\% (m/m) PEO $2.000 \mathrm{~g} \mathrm{~mol}^{-1} \mathrm{e}$ $40,0 \%(\mathrm{~m} / \mathrm{m})$ de $\left(\mathrm{NH}_{4}\right)_{2} \mathrm{SO}_{4}$ verificou-se que os compostos $\mathrm{NH}_{4} \mathrm{~F}$ ou $\mathrm{NH}_{4} \mathrm{Cl}$ não afetam o coeficiente de distribuição destes íons ${ }^{76}$. Porém, nestes $\mathrm{SAB}$, a extração do íon $\mathrm{Hg}^{2+}$ é efetiva, fornecendo valores de $D_{\mathrm{Hg}}^{\text {máx }} \approx 750$.

Na presença de $\mathrm{NH}_{4} \mathrm{I}$, em concentração de $0,5 \mathrm{~mol} \mathrm{~L}^{-1}$, todos os metais foram extraídos com eficiência $\left(D_{M}>100\right)$ para a fase rica em $\mathrm{PEO}$ na ordem de $\mathrm{Tl}^{+}>\mathrm{Hg}^{2+}>\mathrm{Bi}^{3+}>\mathrm{Cd}^{2+}>\mathrm{Pb}^{2+}$, com $\mathrm{D}_{\mathrm{Hg}} \approx 800$.

A influência da presença de $\mathrm{H}_{2} \mathrm{SO}_{4}$ nas fases dos $\mathrm{SAB}$, sobre a extração de íons metálicos, apresenta um comportamento ainda não explicado. Os íons $\mathrm{Zn}^{2+}, \mathrm{Co}^{2+}, \mathrm{Fe}^{3+}$ e $\mathrm{Mo}^{5+}$ não mostram mudança significativa em seus coeficientes de distribuição à medida que a concentração de ácido sulfúrico aumenta. Entretanto, alguns íons lantanídeos e actinídeos têm seus $\mathrm{D}_{\mathrm{M}}$ modificados com a elevação na concentração deste ácido. $O$ gálio, por ex., tem seu coeficiente de distribuição aumentado de 1 para 25 , quando a concentração do $\mathrm{H}_{2} \mathrm{SO}_{4}$ varia de 0,25 para $1,00 \mathrm{~mol} \mathrm{~L}^{-1}$ na presença do ânion tiocianato.

\section{Extração de íons em SAB na presença de um agente extrator ligado ao polímero}

Complexantes derivados de PEO (PEO-ácido iminodiacético, PEO-tris(carboximetilato)etilenodiamina, PEO-carboximetiladotris(2-aminoetil)amina, PEO-ácido aspártico) são considerados possíveis agentes extratores alternativos para partição dos íons metálicos para a fase polimérica. Isso ocorre porque tais compostos demonstram certa afinidade pelo íon, produzindo um complexo metálico que é extraído para a fase rica em polímero devido à forte afinidade dos complexantes derivados do poli(óxido de etileno) por esta fase $\mathrm{e}^{77-79}$.

Aguinaga-Diaz et al. ${ }^{80}$ utilizaram um SAB, formado por $14,0 \%$ $(\mathrm{m} / \mathrm{m})$ PEO $8.000 \mathrm{~g} \mathrm{~mol}^{-1}$ e $8,0 \%(\mathrm{~m} / \mathrm{m}) \mathrm{Na}_{2} \mathrm{SO}_{4}$, para estudar o coeficiente de distribuição dos íons $\mathrm{Cu}$ (II), $\mathrm{Ni}$ (II) e $\mathrm{Co}$ (II), empregando cada um dos três complexantes citados acima e derivados do PEO, obtendo $\mathrm{D}_{\mathrm{M}}>1$. Vale ressaltar que esses derivados de PEO ainda não estão disponíveis comercialmente, sendo necessário sintetizá-los.

\section{CONCLUSÃO}

O SAB tem se mostrado um método de extração eficiente e até seletivo para certos íons, sendo possível sua aplicação a amostras reais. Sua utilização como um método alternativo, em substituição àqueles que empregam solventes orgânicos, é completamente viável e vantajoso devido a sua não-toxicidade. Nos últimos anos, tem sido observado aumento no interesse em medidas do valor de $\mathrm{D}_{\mathrm{M}}$ de vários íons. 
$\mathrm{Na}$ ausência de um agente extrator adicionado no SAB, o ânion $\mathrm{TcO}_{4}-$ mostrou ser o único extraído com sucesso para a fase rica em $\mathrm{PEO}$ em vários $\mathrm{SAB}$. Assim, para a maioria dos íons metálicos estudados, uma extração significativa para a fase rica em polímero somente foi atingida na presença de um extrator. Destaca-se o uso dos complexantes AX, AC e arsenazo III para a extração de actinídeos e lantanídeos, éteres coroa para os metais dos grupos I e II e ânions de haletos, tiocianato e derivados de PEO para extração de metais de transição.

Apesar do progresso alcançado mediante o aumento no número de estudos sobre a partição de íons metálicos em SAB sendo alguns deles patenteados ${ }^{81,82}$, ainda são necessárias investigações que contribuam para a compreensão dos parâmetros que governam a partição desses íons metálicos nos $\mathrm{SAB}$, a fim de otimizar o potencial extrator e a aplicabilidade deste sistema.

\section{AGRADECIMENTOS}

À CAPES pela bolsa de mestrado de F. J. Paggioli e à FAPEMIG pelo auxílio financeiro.

\section{REFERÊNCIAS}

1. Nameroff, T. J.; Garant, R. J.; Albert, M. B.; Res. Policy 2004, 33, 959.

2. Pedersen, B. S.; Rasmussen, K. E.; Halvorsen, T. G.; J. Chromatogr., A 2000, 902, 91 .

3. da Silva, M. C. H.; da Silva L. H. M.; Paggioli, F. J.; Anal. Sci. 2005, 21, 933.

4. Lenardão, E. J.; Freitag, R. A.; Dabdoub, M. J.; Batista, A. C. F.; Silveira, C. C.; Quim. Nova 2003, 26, 123.

5. Klomklao, S.; Benjakul, S.; Visessanguan, W.; Simpson, B. K.; Kishmura, H.; Process Biochem. 2005, 40, 3061.

6. Giraldo-Zuniga, A. D.; Coimbra, J. S. R.; Minim, L. A.; Rojas, E. E. G.; J. Food Eng. 2006, 72, 302.

7. Terreni, M.; Ubiali, D.; Pagani, G.; Hernandez-Justiz, O.; FernandezLafuente, R.; Guisan, J. M.; Enzyme Microb. Technol. 2005, 36, 672.

8. Esmanhoto, E.; Kilikian, B. V.; J. Chromatogr., B: Anal. Technol. Biomed. Life Sci. 2004, 807, 139.

9. Rito-Palomares, M.; J. Microbiol. Biotechnol. 2002, 12, 535.

10. Griffin, S. T.; Spear, S. K.; Rogers, R. D.; J. Chromatogr., B: Anal. Technol. Biomed. Life Sci. 2004, 807, 151.

11. Zhi, W. B.; Song, J. N.; Ouyang, F.; Bi, J. X.; J. Biotechnol. 2005, 118, 157.

12. Kepka, C.; Collet, E.; Roos, F.; Tjerneld, F.; Veide, A.; J. Chromatogr., A 2005, 1075, 33.

13. Haghtalab, A.; Mokhtarani, B.; J. Chem. Thermodyn. 2005, 37, 289.

14. Antov, M. G.; Carbohydr. Polym. 2004, 56, 295.

15. Oliveira, L. A.; Sarubbo, L. A.; Porto, A. L. F.; Campos-Takaki, G. M.; Tambourgi, E. B.; Process Biochem. 2002, 38, 693.

16. Haraguchi, L. H.; Mohamed, R. S.; Loh, W.; Pessoa, P. A.; Fluid Phase Equilibr. 2004, 215, 1.

17. Rogers, R. D.; Bond; A. H.; Bauer, C. B.; Sep. Sci. Technol. 1993, $28,139$.

18. Helfrich, M. R.; El-Kouedi, M.; Etherton, M. R.; Keating, C. D.; Langmuir 2005, 21, 8478.

19. Zeng, X.; Quaye, J.; Osseo-Asare, K.; Colloids Surf., A 2004, 246, 135.

20. Zeng, X.; Osseo-Asare, K.; Colloids Surf., A 2003, 226, 45.

21. Beijerinck, M. W.; Zbl. Bakt. II Natur 1896, 627, 698.

22. Beijerinck, M. W.; Kolloid-Z. Polym. 1910, 7, 16.

23. Zvarova, T. I.; Shkinev, V. M.; Vorobeva, G. A.; Spivakov, B. Y.; Zolotov, Y. A.; Mikrochim. Acta 1984, 3, 449.

24. Zaslavsky, B. Y.; Aqueous Two Phase Partitioning: Physical Chemistry and Bioanalytical Applications; Marcel Dekker: New York, 1995.

25. Ishii, K.; Tanaka, Y.; Hata, K.; Goto, M.; Saitoh, K.; Minamisawa, H.; Shibukawa, M.; Bunseki Kagaku 2004, 53, 911.

26. da Silva, L. H. M.; Loh, W.; Quim. Nova, no prelo.

27. da Silva, L. H. M.; da Silva, M. D. H.; Mesquita, A. F.; do Nascimento, K. S.; Coimbra, J. S. R.; Minim, L. A.; J. Chem. Eng. Data 2005, 50, 1457.

28. Abraham, M. H.; Zissimos, A. M.; Huddleston, J. G.; Willauer, H. D.; Rogers, R. D.; Acree, W. E.; Ind. Eng. Chem. Res. 2003, 42, 413.

29. Rogers, R. D.; Bond, A. H.; Bauer, C. B.; Zhang, J.; Jezl, M. L.; Roden, D. M.; Rein, S. D.; Chomko, R. R. Em Aqueous Biphasic Separations: Biomolecules to Metal Ion; Rogers, R. D.; Eiteman, M. A., eds.; Plenum Press: London, 1995.
30. Rogers, R. D.; Bond, A. H.; Bauer, C. B.; Zhang, J.; Griffin, S. T.; J. Chromatogr., B: Anal. Technol. Biomed. Life Sci. 1996, 680, 221.

31. Shibukawa, M.; Nakayam, N.; Hayashi, T.; Shibuya, D.; Endo, Y.; Kawamura, S.; Anal Chim. Acta 2001, 427, 293.

32. Koch, H. F.; Shen, J.; Roundhill, D. M.; Sep. Sci. Technol. 2000, 35, 623.

33. da Silva, L. H. M.; Meirelles, A. J. A.; Carbohydr. Polym. 2001, 46, 267.

34. Rito-Palomares, M.; Negrete, A.; Miranda, L.; Flores, C.; Galindo, E.; Serrano-Carreon, L.; Enzyme Microb. Technol. 2001, 28, 625.

35. Jiang, J. W.; Prausnitz, J. M.; J. Phys. Chem. B 2000, 104, 7197.

36. Sarubbo, L. A.; Oliveira, L. A.; Porto, A. L. F.; Duarte, H. S.; CarneiroLeao, A. M. A.; Lima, J. L.; Campos-Takaki, G. M.; Tambourgi, E. B.; J. Chromatogr., B: Anal. Technol. Biomed. Life Sci. 2000, 743, 79.

37. Shkinev, V. M.; Molochnikova, N. P.; Spivakov, B. Y.; Myasoedov, B. F. Em ref. 29.

38. Nifanteva, T. I.; Shkinev, V. M.; Spivakov, B. T.; Zolotov, Y. A.; Anal. Chem. USSR 1989, 44, 1105.

39. Rogers, R. D.; Zhang, J.; J. Chromatogr., B: Anal. Technol. Biomed. Life Sci. 1996, 680, 231.

40. Zafarani-Moattar, M. T.; Sadeghi, R.; J. Chem. Eng. Data 2005, $50,947$.

41. Rogers, R. D.; Griffin, S. T.; J. Chromatogr., B: Anal. Technol. Biomed. Life Sci. 1998, 711, 277.

42. Rogers, R. D.; Bauer, C. B.; J. Chromatogr., B: Anal. Technol. Biomed. Life Sci. 1996, 680, 237

43. Chen, J.; Spear, S. K.; Huddleston, J. G.; Rogers, R. D.; Green Chem. 2005, $7,64$.

44. Tada, E. D.; Loh, W.; Pessoa, P. D.; Fluid Phase Equilibr. 2005, 231, 250.

45. Li, L.; He, C. Y.; Li, S. H.; Liu, F.; Su, S.; Kong, X. X.; Li, N.; Li, K. A.; Chin. J. Chem. 2004, 22, 1313.

46. Spivakov, B. Y.; Nifanteva, T. I.; Shkinev, V. M. Em ref. 29.

47. Rogers, R. D.; Bauer, C. B.; Bond, A. H.; J. Alloys Compd. 1994, 213, 305 .

48. Guzmán, R.; Téllez, C. M. Em ref. 29.

49. Nair, A. G. C.; Das, S. K.; Deshmukh, S. M.; Prakash, S.; Radiochim. Acta 1992, 57, 29.

50. Rogers, R. D.; Bond; A. H.; Bauer, C. B.; Zhang, J.; Appl. Radiat. Isot. 1996, 47, 497.

51. Rogers, R. D.; Zhang, J. H.; Bond, A. H.; Bauer, C. B.; Jezl, M. L.; Roden, D. M.; Solvent Extr. Ion Exch. 1995, 13, 665.

52. Rogers, R. D.; Bond, A. H.; Griffin, S. T.; Horwitz, E. P.; Solvent Extr. Ion Exch. 1996, 14, 919.

53. Rogers, R. D.; Bond, A. H.; Bauer, C. B.; Zhang, J. H.; Rein, S. D.; Chomko, R. R.; Roden, D. M.; Solvent Extr. Ion Exch. 1995, 13, 689.

54. Tubio, G.; Nerli, B.; Pico, G.; J. Chromatogr., B: Anal. Technol. Biomed. Life Sci. 2004, 799, 293.

55. Rogers, R. D.; Bond, A. H.; Bauer, C. B.; Pure Appl. Chem. 1993, 65, 567.

56. Alvarez, A.; Gasco, C.; Navarro, N.; Anton, M. P.; Sancho, C.; J. Radioanal. Nucl. Chem. 2005, 265, 383.

57. Rogers, R. D.; Zhang, J. H.; Griffin, S. T.; Sep. Sci. Technol. 1997, 32, 699.

58. Spea, S. K.; Griffin, S. T.; Huddleston, J. G.; Rogers, R. D.; Ind. Eng. Chem. Res. 2000, 39, 3173.

59. Rogers, R. D.; Zhang, J. H.; Bond, A. H.; Horwitz, E. P.; Sep. Sci. Technol. 1997, 32, 867.

60. Molochnikova, N. P.; Shkinev, V. M.; Myasoedov, B. F.; Solvent Extr. Ion Exch. 1992, 10, 697.

61. Pawaskar, C. S.; Mohapatra, P. K.; Manchanda, V. K.; Radiochim. Acta 1998, $81,11$.

62. Pawaskar, C. S.; Mohapatra, P. K.; Manchanda, V. K.; J. Radioanal. Nucl. Chem. 1999, 242, 627.

63. Shkinev, V. M.; Molochnikova, N. P.; Zavarova, T. I.; Spivakov, B. Y.; Myasoedov, B. F.; Zolotov, Y. A. Em Proceedings of the International Symposium on Actinide and Lanthanide Separations; Choppin, G. R.; Navratil, J. D.; Schulz, W. W., eds.; World Scientific: Singapore, 1985.

64. Shkinev, V. M.; Molochnikova, N. P.; Frenkel, V. Ya.; Spivakov, B. Y.; Myasoedov, B. F.; Zolotov, Y. A.; Radiokhimiya 1987, 29, 330.

65. Shkinev, V. M.; Molochnikova, N. P.; Spivakov, B. Y.; Myasoedov, B. F.; Zolotov, Y. A.; Radiokhimiya 1988, 30, 60.

66. Myasoedov, B. F.; Karalova, Z. K.; Bukinaa, T. I.; Lavrinovich, E. A.; Solvent Extr. Ion Exch. 1988, 6, 1109.

67. Rogers, R. D.; Bauer, C. B.; Bond, A. H.; Sep. Sci. Technol. 1995, 30, 1203.

68. Huddleston, J. G.; Willauer, H. D.; Boaz, K. R.; Rogers, R. D.; J. Chromatogr., B: Anal. Technol. Biomed. Life Sci. 1996, 34, 1069.

69. Moody, M. L.; Willauer, H. D.; Griffin, S. T.; Huddleston, J. G.; Rogers, R. D.; Ind. Eng. Chem. Res. 2005, 44, 3749.

70. Shkinev, V. M.; Molochnikova, N. P.; Zavarova, T. I.; Spivakov, B. Y.; Myasoedov, B. F.; Zolotov, Y. A.; J. Radioanal. Nucl. Chem. 1985, 88, 115. 
71. Molochnikova, N. P.; Shkinev, V. M.; Myasoedov, B. F.; Radiokhimiya 1995, 37, 477 .

72. Rogers, R. D.; Bauer, C. B.; J. Radioanal. Nucl. Chem. 1996, $208,153$.

73. Du, H. S.; Wood, D. J.; Elshani, S.; Wai, C. M.; Talanta 1993, 40, 173.

74. Luo, H. M.; Dai, S.; Bonnesen, P. V.; Buchanan, A. C.; Holbrey, J. D. Bridges, N. J.; Rogers, R. D.; Anal. Chem. 2004, 76, 3078.

75. Shibukawa, M.; Matsuura, K.; Shinozuka, Y.; Mizuno, S.; Oguma, K.; Anal Sci. 2000, 16, 1039.

76. Rogers, R. D.; Bond, A. H.; Bauer, C. B.; Abstracts of Paper of the American Chemical Society 1992, 203, 88-IEC Part 2.
77. Lin, D. Q.; Yao, S. J.; Mei, L. H.; Zhu, Z. Q.; Chin. J. Chem. Eng. 2000, 8,310 .

78. Laboureau, E.; Vijayalakshmi, M. A.; J. Mol. Recognit. 1997, 10, 262

79. Laboureau, E.; Capiod, J. C.; Dessaint, C.; Prin, L.; Vijayalakshmi, M. A.; J. Chromatogr., B: Anal. Technol. Biomed. Life Sci. 1996, 680, 189.

80. Aguinaga-Diaz, P. A.; Guzman, R. Z.; Sep. Sci. Technol. 1996, 31, 1483.

81. Guinn Martin, R.; US pat. 19970861587 19970522, 1999.

82. Shibukawa M.; JP pat. $2003154201-A, 2003$. 